\title{
Parent-child behavioural patterns related to pre-schoolers' overweight/obesity
}

\author{
Dagmar Sigmundová1,*, Erik Sigmund ${ }^{1}$, Petr Badura ${ }^{1}$, Jana Vokáčová1, Daniel Klein ${ }^{2}$, \\ and Jens Bucksch ${ }^{3}$
}

${ }^{1}$ Faculty of Physical Culture, Palacký University Olomouc, Olomouc, Czech Republic; ${ }^{2}$ Faculty of Science, Pavol Josef Šafárik University in Košice, Košice, Slovak Republic; and ${ }^{3}$ Department of Natural and Human Sciences, Prevention and Health Promotion, Heidelberg University of Education, Heidelberg, Germany

Copyright: @ 2017 D. Sigmundová et al. This is an open access article licensed under the Creative Commons Attribution License (http://creativecommons.org/licenses/by/4.0/).

\begin{abstract}
Background: The increasing prevalence of childhood obesity is a global concern. Although childhood obesity has grown as a result of a complex array of interactions among multiple behavioural, biological, and environmental factors, excessive screen time (ST) and low levels of physical activity (PA) are often discussed as causal factors. Therefore, it is beneficial to identify risky family behavioural patterns contributing to the increasing prevalence of obesity even in pre-schoolers. Objective: The main aim of the study was to assess whether parental obesity and parent-child behavioural patterns (PA and ST) affect the odds of overweight/obesity in 4-to-7-year-old preschool children. Methods: We analysed seven-day PA and ST behaviour among families with pre-schoolers that included 194 preschool children (88 girls and 106 boys) and their parents (165 mothers and 111 fathers). PA was monitored by means of unsealed Yamax pedometers for at least eight hours a day over seven consecutive days during spring (April/May) and autumn (September/October) of 2015. ST was recorded by parents on family log book sheets. To assess the odds of parents' obesity and PA/ST variables in relation to child overweight/obesity, a logistic regression (backward method) was used. Results: Most children $(n=157)$ reported normal weight ( 87 boys, 70 girls), 37 children were overweight or obese (19 boys, 18 girls). Children's excessive ST (> 1 hour/day) (OR: 5.65/33.19 on weekdays/weekends), mothers' obesity (OR: 13.80/28.84 on weekdays/weekends), mothers' excessive ST ( $>2$ hours/ day) (OR: 32.46 at weekends), and children's male gender (OR: 38.69 at weekends) were significantly $(p<.05)$ associated with higher odds of overweight/obesity in the preschool children. Conclusions: Uncovering parent-child behavioural patterns provides insight into the lifestyle of families with pre-schoolers and is a source of valuable information for designing and implementing family-based intervention programmes aimed at reducing obesity. Especially, weekends provide a suitable space for the implementation of joint family programmes aimed at reducing sedentary behaviour.
\end{abstract}

Keywords: obesity, mother, screen time, physical activity, weekdays, weekends

\section{Introduction}

The worldwide prevalence of overweight/obesity among preschool children increased from $4.2 \%$ in 1990 to $6.7 \%$ in 2010 , with the expected representation of overweight/obesity accounting for $9.1 \%$ or $\approx 60$ million in 2020, and the estimate is even higher (14.1\%) in developed countries (de Onis, Blössner, \& Borghi, 2010), despite the differences in the prevalence of overweight/obesity among pre-schoolers from different continents and countries (de Onis et al., 2010;

\footnotetext{
* Address for correspondence: Dagmar Sigmundová, Institute of Active Lifestyle, Faculty of Physical Culture, Palacký University Olomouc, třída Míru 117, 771 11, Olomouc, Czech Republic. E-mail: dagmar.sigmundova@upol.cz
}

Katzmarzyk et al., 2013). The epidemic of childhood obesity could be explained by a complex interaction of multiple biological, behavioural, and environmental factors that lead to a long-term positive energy balance between food intake and expenditure (Katzmarzyk et al., 2013). Besides the risk of the early onset of metabolic syndrome, cardiovascular disease, type 2 diabetes, and musculoskeletal disorders, overweight/obesity in pre-schoolers also tends to track significantly into adulthood (Whitaker, Wright, Pepe, Seidel, \& Dietz, 1997).

Parents play an essential role in the mental, physical, and social development of their children (Bandura, 2001), especially pre-schoolers. The uncovered correlates of overweight/obesity of preschool children 
include parental obesity (Parikka et al., 2015; Whitaker et al., 1997), especially in mothers (Durmuş et al., 2013; Kitsantas \& Gaffney, 2010), or both parents being obese (Durmuş et al., 2013; Parikka et al., 2015), high birth weight of children (Kitsantas \& Gaffney, 2010), non-married mothers belonging to low socioeconomic groups (Kitsantas \& Gaffney, 2010), low levels of education of parents (Parikka et al., 2015), low levels of physical activity (PA), and excessive television viewing (Mendoza, Zimmerman, \& Christakis, 2007; te Velde et al., 2012).

From the above-mentioned correlates of overweight/obesity among pre-schoolers, the objective measurement of overweight/obesity in parents and children is methodologically relatively simple and accurate, while the measurement of free-living PA, sedentary behaviour, or eating habits is more complicated and methodologically diverse (Kelly, Fitzsimons, \& Baker, 2016). Previous comprehensive studies of energy balance-related behaviours in preschool children from six European countries (Belgium, Bulgaria, Germany, Greece, Poland, and Spain) (Cardon et al., 2016; De Craemer, Lateva, et al., 2015; van Stralen et al., 2012) used objectively measured overweight status, PA measured by pedometers, and parents' proxy-reported questionnaires for assessing the sedentary behaviour and dietary habits of pre-schoolers (Cardon et al., 2016). However, the aforementioned studies (Cardon et al., 2016; De Craemer, Lateva, et al., 2015) did not use a strict inclusion criterion for PA regarding the number of days of pedometer-monitored PA of pre-schoolers (e.g. a minimum of two weekdays and one weekend day). Consistently with the results of numerous studies (Aadland \& Johannessen, 2015; Corder, Ekelund, Steele, Wareham, \& Brage, 2008; Rowe, Mahar, Raedeke, \& Lore, 2004), we recommend at least seven consecutive days of monitoring after a familiarization day including both weekend days for accurate and reliable estimates of the habitual PA behaviour of pre-schoolers to reveal weekday-weekend patterns of PA behaviour.

This study explores in greater depth the lack of existing studies on parent-child weekday-weekend behavioural patterns related to overweight/obesity among pre-schoolers from a Central European country and complements the results for health-related behaviours in normal weight and overweight/obese pre-schoolers of a large pan-European sample.

The main aim of the study was to determine the family variables on weekdays and at weekends that increase the odds of overweight/obesity among 4-to-7-year-old preschool children during a full one-week monitoring of their own and their parents' PA and screen time (ST). The specific objectives were:

- to describe the level of daily PA in relation to gender, overweight/obesity, and weekdays/weekend days among Czech preschool children;

- to compare the amount of ST on weekdays and at weekends between Czech families with pre-schoolers with normal body weight and families with overweight/obese pre-schoolers;

- to describe the prevalence of overweight and obesity in Czech families by gender, in relation to PA and ST.

\section{Methods}

\section{Ethics}

The Ethical Committee of the Faculty of Physical Culture, Palacký University Olomouc approved the study (ref. no. 57/2014) on 10 December 2014. The objectives, content, and the protocol of the research were thoroughly explained to the parents of the children involved in the study and teachers and kindergarten employees at a joint meeting with the authors of the project in each of the kindergartens. They received instructions regarding the way the pedometer should be used and the process of recording the monitored values into a family log book. The parents were also informed that before the start of the one-week monitoring of PA and ST they would be asked to record the basic anthropometric parameters of all the family members participating in the research. The participation in this study was voluntary and unpaid; parents were required to provide written informed consent, if they and their children wished to participate in this study. Each participant who completed the one-week monitoring of PA and ST received individual feedback on the results, together with recommendations for health-promoting PA.

\section{Selection of participants and data inclusion criteria}

A total of 296 preschool children (141 girls and 155 boys) from 10 randomly selected kindergartens, together with their parents (234 mothers and 181 fathers), were asked to participate in the study. The kindergartens were selected from all four Moravian regions of the Czech Republic and included schools from cities, towns, and rural areas. The monitoring of weekly PA and ST was conducted during April/May and September/October 2015. The consecutive 7-day monitoring of PA and ST was completed by more than $75 \%$ of the participants (223 children and 313 parents). The data of 29 children and 37 parents was not included in the analyses because of missing data on 
body weight, height, or age or other exclusion criteria (i.e. an absence of more than one day of the child from kindergarten or insufficient time $[<8$ hours] spent daily wearing the pedometer). In accordance with the recommendations of previous studies (Aadland \& Johannessen, 2015; Corder et al., 2008; Rowe et al., 2004), the final analyses included data from parentchild dyads (both parents and child $n=92$, mother and child $n=165$, and father and child $n=112$ ). Table 1 depicts the basic anthropometric characteristics of the participants split into two groups based on the level of body weight of the pre-schoolers.

\section{Assessment of physical activity, screen time, and anthro- pometric parameters \\ Instruments}

The parents and their children wore an unsealed Yamax Digiwalker SW-200 pedometer (Yamax Corporation, Tokyo, Japan). This kind of Yamax pedometer does not need to be set up individually (e.g. entering the user's height or weight or step length) before use. The reasonable reliability and validity of the hip-worn Yamax Digiwalker SW-200 step count measurement in 3-5-year-old children was confirmed by concurrent direct observation using the Children's Activity Rating Scale (Oliver, Schofield, Kolt, \& Schluter, 2007).

Daily step count values and the duration of ST were recorded by the parents in a family log book. The duration of daily ST behaviour (sitting or lying while watching TV [video, DVD] and sitting or lying in front of a PC [including a notebook, tablet, or smartphone]) was fixed at $10 \mathrm{~min}$. The parents' proxy reports of the time their 5-6-year-old children spent watching TV daily exhibit an acceptable 7-to-14-day test-retest reliability $(I C C=.78, p<.001)$ for capturing sedentary behaviour on regular weekday and weekend days (Salmon, Campbell, \& Crawford, 2006). The parents also reported their own and their children's body weight and height. They were instructed how to measure their own as well as their child's body height and weight at

Table 1

Descriptive characteristics of families with overweight/obese pre-schoolers and pre-schoolers with normal body weight

\begin{tabular}{|c|c|c|c|c|}
\hline & \multicolumn{2}{|c|}{$\begin{array}{c}\text { Families with } \\
{\text { overweight } / \text { obese }^{\#} \text { pre-schoolers }}^{\text {ons }}\end{array}$} & \multicolumn{2}{|c|}{$\begin{array}{l}\text { Families with } \\
\text { pre-schoolers with normal body weight }\end{array}$} \\
\hline & $M$ & $S D$ & $M$ & $S D$ \\
\hline Children & \multicolumn{2}{|c|}{$n=37$} & \multicolumn{2}{|c|}{$n=157$} \\
\hline Gender & \multicolumn{2}{|c|}{$49 \%$ female } & \multicolumn{2}{|c|}{$45 \%$ female } \\
\hline Age (years) & 5.55 & 0.86 & 5.62 & 0.85 \\
\hline Body height $(\mathrm{cm})$ & 116.19 & 9.76 & 117.13 & 8.88 \\
\hline Body weight (kg) & 25.22 & 4.61 & 20.01 & 2.99 \\
\hline BMI $\left(\mathrm{kg} / \mathrm{m}^{2}\right)$ & 18.55 & 1.35 & 14.58 & 1.50 \\
\hline \multicolumn{5}{|l|}{ Overweight/obese } \\
\hline Girls & \multicolumn{2}{|c|}{$11.7 \% / 9.1 \%$} & \multicolumn{2}{|c|}{-} \\
\hline Boys & \multicolumn{2}{|c|}{$8.5 \% / 9.4 \%$} & \multicolumn{2}{|c|}{-} \\
\hline Mothers & \multicolumn{2}{|c|}{$n=30$} & \multicolumn{2}{|c|}{$n=135$} \\
\hline Age (years) & 34.53 & 4.76 & 36.39 & 4.12 \\
\hline Body height $(\mathrm{cm})$ & 167.90 & 6.10 & 167.58 & 6.20 \\
\hline Body weight (kg) & 74.07 & 13.29 & 66.46 & 10.85 \\
\hline BMI $\left(\mathrm{kg} / \mathrm{m}^{2}\right)$ & 26.28 & 4.64 & 23.69 & 3.87 \\
\hline Overweight/obese ${ }^{\S}$ & \multicolumn{2}{|c|}{$30.0 \% / 26.7 \%$} & \multicolumn{2}{|c|}{$24.1 \% / 7.5 \%$} \\
\hline Fathers & \multicolumn{2}{|c|}{$n=18$} & \multicolumn{2}{|c|}{$n=93$} \\
\hline Age (years) & 37.94 & 4.02 & 38.85 & 5.07 \\
\hline Body height $(\mathrm{cm})$ & 180.53 & 6.60 & 180.46 & 6.91 \\
\hline Body weight (kg) & 81.82 & 8.93 & 85.45 & 12.73 \\
\hline BMI $\left(\mathrm{kg} / \mathrm{m}^{2}\right)$ & 25.13 & 2.69 & 26.18 & 3.36 \\
\hline Overweight/obese ${ }^{\S}$ & \multicolumn{2}{|c|}{$35.3 \% / 5.9 \%$} & \multicolumn{2}{|c|}{$55.4 \% / 10.9 \%$} \\
\hline
\end{tabular}

Note. $\quad$ BMI = body mass index. ${ }^{\#}$ Overweight/obesity in children older than five was classified using the World Health Organization (WHO) percentile BMI charts (WHO, 2007) and in children aged less than five using the corresponding age-differentiated BMI charts (WHO Multicentre Growth Reference Study Group \& de Onis, 2006); $\$$ Overweight and obesity in parents refer to a BMI from $25 \mathrm{~kg} / \mathrm{m}^{2}$ to $29.9 \mathrm{~kg} / \mathrm{m}^{2}$ and greater than or equal to $30 \mathrm{~kg} / \mathrm{m}^{2}$, respectively (WHO, 2014). 
home (height - standing still on a flat floor, against the wall and without shoes, values rounded to the nearest $0.5 \mathrm{~cm}$, weight - in underwear, values rounded to the nearest $0.5 \mathrm{~kg}$ ). The parents' home measurement of the body weight/height of their 3-7-year-old children was verified by comparison with laboratory measurements (weight: $I C C=.94$, height: $I C C=.95$, BMI: $I C C=.67$ ) without significant difference between the proportion of overweight/obese pre-schoolers on the basis of the home and laboratory measurements $\left(8 \% / 2.9 \%_{\text {Hоме }}\right.$ vs. $11.7 \% / 3.8 \%_{\text {LABORATORY }}$, difference $=3.7 \% / 1.3 \%$, $p=.180 / .625$ ) (Huybrechts et al., 2011). The chronological age of all the family members was calculated from their date of birth until the starting day of the PA/ST monitoring.

\section{Procedure}

The participants wore a pedometer on their right hip for a continuous period of eight days. The values from the first day of monitoring were not included in the final analyses because of insufficient time spent wearing the pedometer and because of the novelty of wearing it, which might have affected the level of the participants' PA (Rowe et al., 2004). Both the children and their parents were instructed to wear the pedometer throughout the whole day except when bathing, showering, dressing, and during personal hygiene. Every morning after their personal hygiene, the parents reset the pedometers, attached them to the right hip (of their children and their own) and recorded the time of resetting in the family log book (Sigmund, Badura, Vokacova, \& Sigmundová, 2016; Sigmundová et al., 2016). In the evening, the parents removed the pedometers and, together with their children, recorded the time and daily step count (PA indicator) of all the participating family members in the log book. In addition to the time and step count, at home in the evening the parents recorded the daily duration of ST behaviour in the family log book.

Before the start of the one-week monitoring of PA and ST, the parents were asked to record the basic information and anthropometric parameters of the family members (date of birth, gender, body height, and weight) in the family log book.

\section{Data analysis and statistics}

The data was analysed using the SPSS software (Version 22; IBM SPSS, Chicago, IL, USA). The BMI was computed as the body weight $(\mathrm{kg})$ divided by body height (m) squared. Age-specific cut-off points, according to the WHO (WHO Multicentre Growth Reference Study Group \& de Onis, 2006; WHO, 2007), were used to define the prevalence of overweight/obesity. Overweight or obesity in children represents a BMI from the 85 th to 97 th or greater than the 97 th percentile of the WHO growth charts (WHO Multicentre Growth Reference Study Group \& de Onis, 2006; WHO, 2007). Overweight/obesity in parents represents a BMI greater than $25 \mathrm{~kg} / \mathrm{m}^{2}$ (WHO, 2014).

The step count data for the seven days was reviewed to check for missing and extreme values. If step counts were recorded during four weekdays, data for the one missing weekday was imputed on the basis of the participant's personal mean scores. Those participants whose step count data was missing for more than one day were excluded from the analysis. The daily step count variable represented the mean difference between morning (pedometer turned on) and evening (pedometer turned off) step counts on the days of the week that were monitored. Daily step count values below 1,000 or exceeding 30,000 were truncated to these recommended limit values, respectively (Craig, Cameron, \& TudorLocke, 2013; Rowe et al., 2004), and included in the analyses. The mean daily step count was calculated for each person separately on the basis of his/her number of steps recorded on five weekdays and two weekend days. The daily step count recommendation for preschool children represents a value of 11,500 steps/day (De Craemer, De Decker, et al., 2015) and for adults it is a value of 10,000 steps/day (Tudor-Locke et al., 2011).

The daily ST variable represented the sum of the time spent sitting and lying while watching TV (DVD, video) and sitting and lying in front of a PC (notebook, tablet, smartphone). An excessive ST for children was defined as more than one hour per day (Australian Government, Department of Health and Ageing, 2013) and for adults as more than two hours per day (Tremblay, Colley, Saunders, Healy, \& Owen, 2010). The data (step counts, screen time) was analysed in total for all the kindergartens because the two-step cluster analysis found no indication for clustering by kindergarten or season.

The differences between the boys' and girls' step counts and ST in the families with pre-schoolers with normal body weight and in the families with overweight/obese pre-schoolers were examined by means of an independent $t$-test (2-tailed). The differences between the weekday/weekend step counts and ST in all participants were tested using a paired $t$-test (2-tailed).

To determine the odds of overweight/obesity among the pre-schoolers, a binomial logistic regression analysis for weekdays and weekends separately (backward method) was used, with the overweight or obesity of the preschool children as a dependent variable and the following independent categorical variables: mother's obesity (yes; no), father's obesity (yes; no), father's ST (> 2 hours/day; less), mother's ST (> 2 hours/day; less), children's ST (>1 hour/day; less), children's gender 
(male; female), mother's PA ( $\geq 10,000$ steps/day; less), father's PA ( $\geq 10,000$ steps/day; less), children's PA ( $\geq 11,500$ steps/day; less). The significance level was set to $p<.05$ (2-tailed). The estimate of the standardized difference between the pairs of variables tested was represented as the Cohen's $d$ coefficient. The $d$ values of $0.2,0.5$, and 0.8 can be interpreted as small, medium, and large effects (Cohen, 1988).

\section{Results}

In the sample, 157 children reported normal weight ( 87 boys, 70 girls) and 37 children were overweight or obese (19 boys, 18 girls) (Table 1).

As a result of non-significant differences between the preschool boys and girls in terms of their daily step counts (families with pre-schoolers with normal body weight: $t_{\text {WEEKDAYS }}=1.59, p=.11, d=0.26$; $t_{\text {WEEKENDS }}=1.85, p=.07, d=0.29$; families with overweight/obese pre-schoolers: $t_{\text {WEEKDAYS }}=0.24, p=.81$, $d=0.08 ; t_{\text {WEEKENDS }}=0.92, p=.37, d=0.30$ ) the following weekday-weekend parent-child comparisons of daily step counts were performed together for the boys and girls (Figure 1). In the following figures (Figures 1 and 2) family-based data (i.e. comprising at least a dyad of one child and one parent) is presented.

More than half of the members of families with pre-schoolers with normal body weight met the recommendations for the daily number of steps $(11,500$ steps per day for children and 10,000 steps per day for adults) on weekdays, compared to the families with overweight/obese pre-schoolers (Figure 1). The preschoolers with normal body weight were more physically active than their overweight/obese peers on whole weekdays $(t=2.19, p=.032, d=0.38)$.

Because of non-significant differences between the preschool boys and girls in terms of their daily ST (families with pre-schoolers with normal body weight: $t_{\text {WEEKDAYS }}=1.90, p=.06, d=0.35 ; \quad t_{\text {WEEKENDS }}=0.04$, $p=.97, d=0.01 ;$ families with overweight/obese pre-schoolers: $t_{\text {WEEKDAYS }}=0.54, \quad p=.60, \quad d=0.21$; $t_{\text {WEEKENDS }}=0.44, p=.66, d=0.18$ ), a weekday-weekend parent-child comparison of daily ST was conducted together for the boys and girls (Figure 2). When comparing the weekday-weekend patterns of ST, we found significantly more ST at weekends than on weekdays in all the family members, except for the fathers of

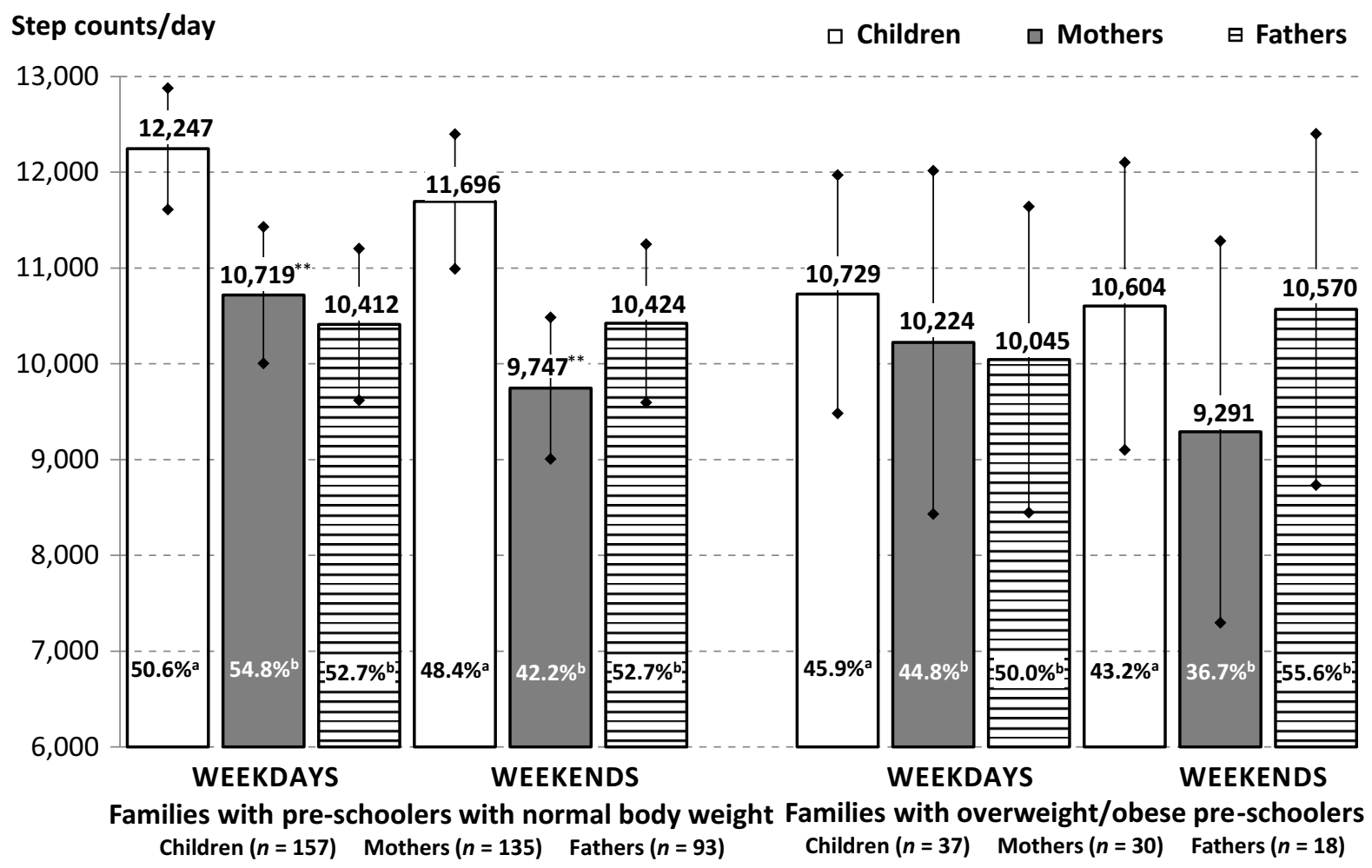

Figure 1. Comparison of parents' and children's pedometer-determined daily step counts (mean and 95\% confidence intervals) on weekdays and at weekends. $\%^{\mathrm{a}}=$ percentages of children who reach the step count recommendation of 11,500 steps per day; $\%^{b}=$ percentages of mothers/fathers who reach the step count recommendation of 10,000 steps per day. The statistical significance of weekday-weekend differences in daily step counts is expressed as ${ }^{* *} p \leq .01$. 


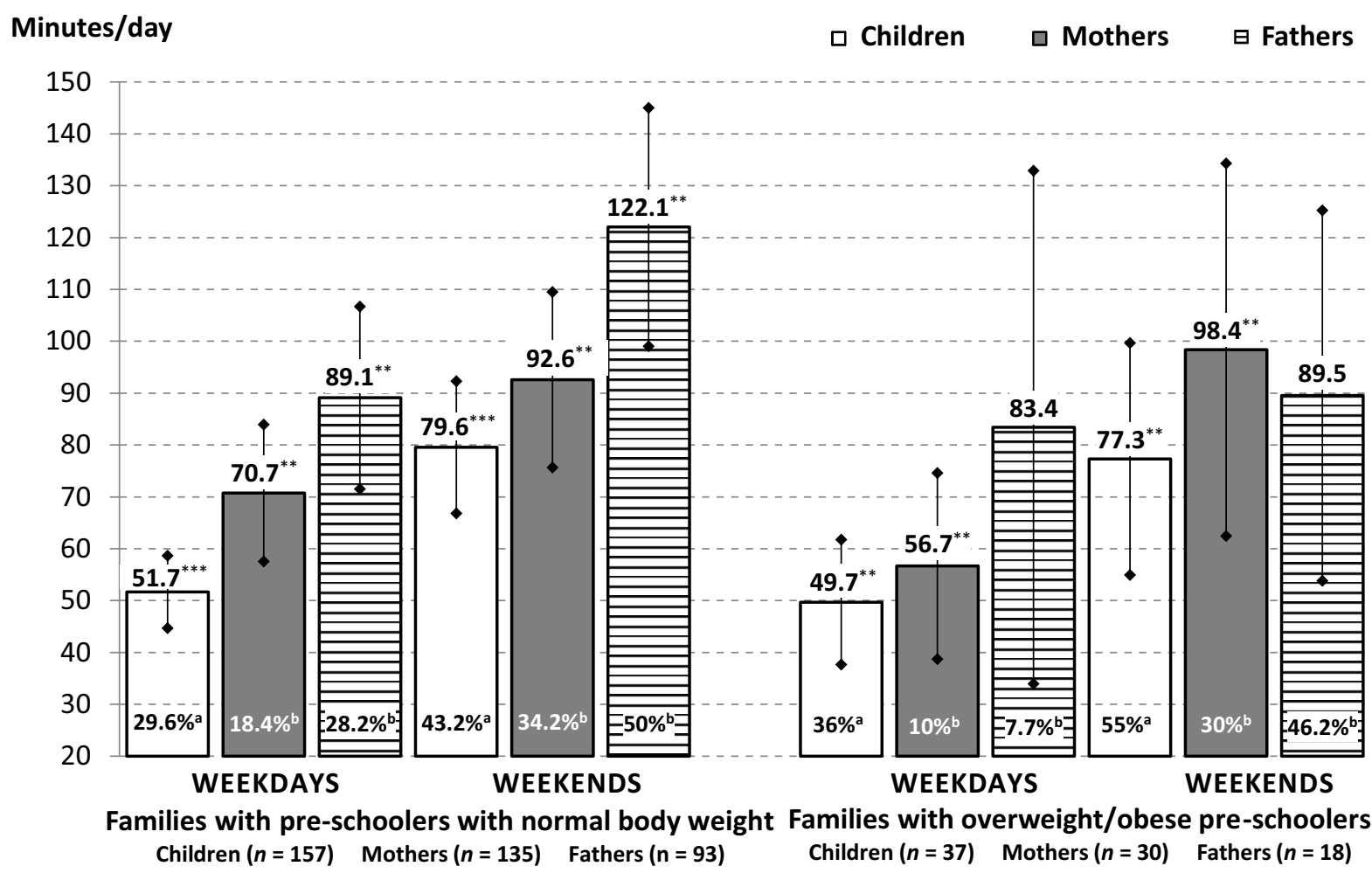

Figure 2. Comparison of parents' and children's logged daily screen time (mean and $95 \%$ confidence intervals) on weekdays and at weekends. $\%^{\mathrm{a}}=$ percentages of children who exceeded 1 hour of screen time per day; $\%^{\mathrm{b}}=$ percentages of mothers/fathers who exceeded 2 hours of screen time per day. The statistical significance of weekday-weekend differences in daily screen time is expressed as ${ }^{* *} p \leq .01,{ }^{* * *} p \leq .001$.

overweight/obese pre-schoolers (Figure 2). The daily ST of the pre-schoolers with normal body weight was almost equivalent to the daily ST in overweight/obese pre-schoolers (Figure 2).

Considering the differences observed in the daily step counts and duration of ST between weekdays and weekends between the families with overweight/obese pre-schoolers and the families with pre-schoolers with normal body weight (Figures 1 and 2), the following examination of the variables associated with the odds of overweight/obesity among pre-schoolers was performed separately for weekdays and weekends (Table 2).

A binomial logistic regression analysis of weekdays (backward method) revealed higher odds of overweight/obesity among the pre-schoolers with obese mothers (OR: 13.80; 95\% CI: [2.20, 86.42]; $p=.005)$ and among the pre-schoolers with excessive ST (> 1 hour/day) (OR: 5.65; 95\% CI: [1.05, 30.54]; $p=.044$ ) (Table 2). The regression model with weekend variables concerning PA/ST suggests that the odds of overweight/obesity among the pre-schoolers are significantly higher with maternal obesity, excessive ST in children, excessive maternal ST (> 2 hours/day), and children's male gender (Table 2). In the regression model with the whole-week variables concerning $\mathrm{PA} / \mathrm{ST}$, the mother's obesity represents a key factor that increases the odds of the preschool children being overweight/obese (OR: 28.01; 95\% CI: [2.74, 286.10]; $p=.005)$. This applies to the crude models as well (Table 2).

\section{Discussion}

The aim of this study was to determine the family variables on weekdays and at weekends that increase the odds of overweight/obesity among preschool children, based on 7-day monitoring of physical activity by means of a pedometer. The pedometer-determined PA in Czech pre-schoolers did not reveal weekdayweekend-related differences in daily step counts. This finding is in contrast to other current European (Cardon et al., 2016; De Craemer, Lateva, et al., 2015) and American studies (Craig et al., 2013). Other studies of weekly PA in pre-schoolers repeatedly observed higher step counts on weekdays than at weekends (Cardon et al., 2016; De Craemer, Lateva, et al., 2015). However, contrary to the results for the PA of pre-schoolers from six European countries (Belgium, Bulgaria, Germany, Greece, Poland, and Spain [Cardon et al., 2016]), the Czech pre-schoolers with normal body weight showed significantly higher step counts on weekdays than the 


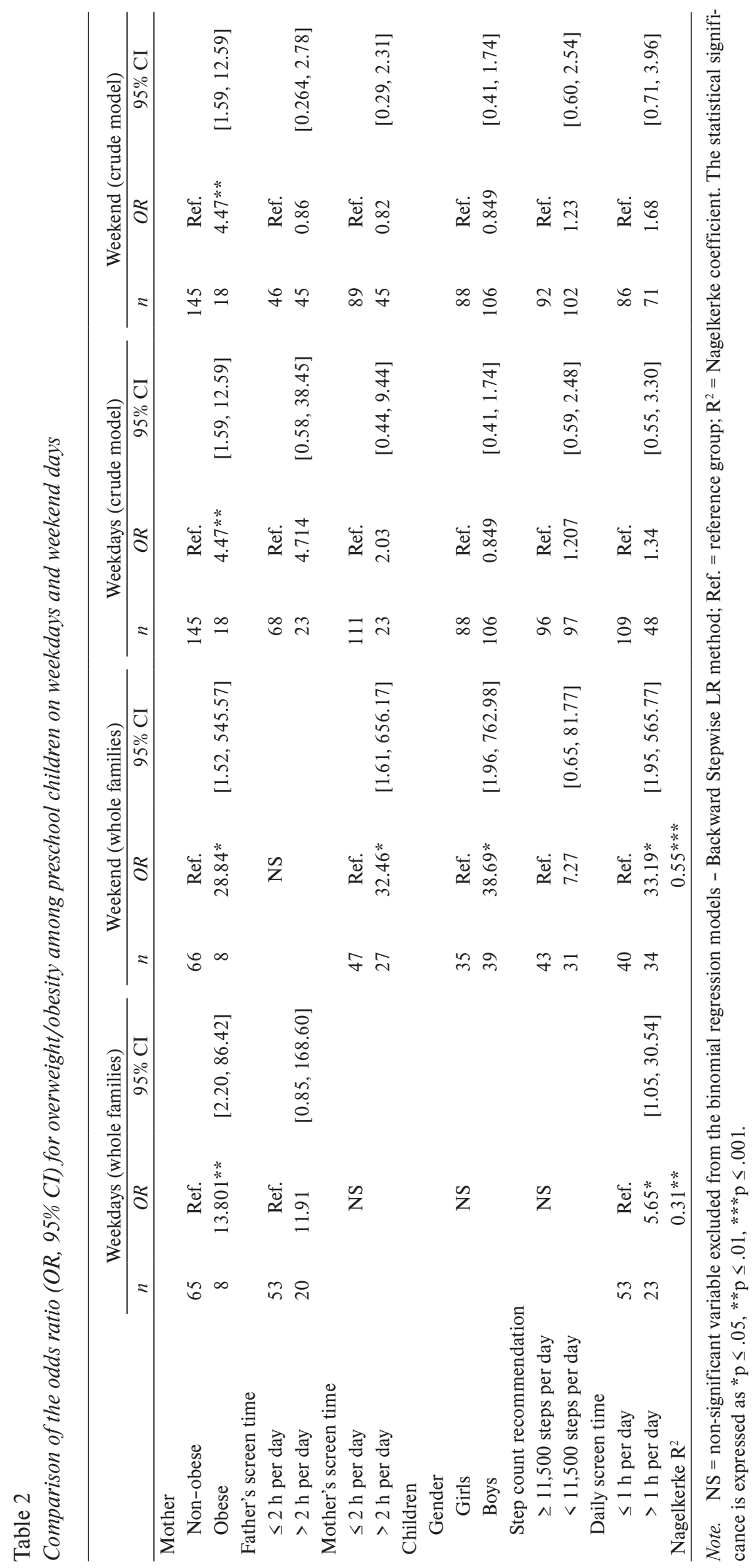


overweight/obese pre-schoolers, but not at weekends. The higher daily step counts on weekdays among preschoolers with normal body weight than among their overweight/obese peers were apparently accumulated as a result of higher step counts in their free time (out-ofkindergarten time), because regarding the sum of time spent at kindergarten there was no significant difference in step counts between the pre-schoolers with normal body weight and those who were overweight/obese (Sigmund, Sigmundová, Badura, Trhlíková, \& Madarasová Gecková, 2016).

Indeed, the proportion of the Czech preschool children with normal or excessive body weight meeting the recommended step count of 11,500 steps a day (De Craemer, De Decker, et al., 2015) on weekdays (normal body weight: $52.9 \%_{\sigma^{*}}$ and $47.8 \%_{9}$; overweight/obese: $42.1 \%_{\sigma^{*}}$ and $50.0 \%_{\rho}$ ) and at weekends (normal body weight: $55.2 \%_{\sigma^{*}}$ and $40.0 \%_{\text {; }}$; overweight/obese: $47.4 \%_{\sigma^{*}}$ and $38.9 \%$ ) was higher than the average proportion of pre-schoolers from six European countries (Belgium, Bulgaria, Germany, Greece, Poland, and Spain) (Cardon et al., 2016) on weekdays (normal body weight: $43.4 \%_{\sigma^{*}}$ and $25.9 \%_{\text {\% }}$; overweight/obese: $38.5 \%_{\sigma^{*}}$ and $26.9 \%_{q}$ ) and at weekends (normal body weight: $32.0 \%$ and $12.0 \%_{\text {q }}$; overweight/obese: $28.8 \%_{\sigma^{*}}$ and $23.6 \%_{q}$ ) (Cardon et al., 2016). The level of the pedometerdetermined PA of the Czech pre-schoolers is in line with the daily step counts of a Canadian sample in 5-6-year-old pre-schoolers (ox: 12,259 steps/day; ㅇ: 11,396 steps/day) (Craig et al., 2013).

We found that the mothers and fathers of the preschool children reveal a different weekday-weekend pattern of PA. While in the mothers we found similar weekday-weekend PA patterns (a decline at weekends) to those found in their children with normal weight or those who were overweight/obese, the fathers showed non-significant changes in their step counts between weekends and weekdays. In accordance with a study (Sigmund, Badura, et al., 2016) that revealed a closer relationship between mother-child weekday-weekend PA than father-child weekday-weekend PA among pre-schoolers, a similar weekday-weekend pattern of pedometer-determined PA in the mothers and their preschool children seems to be logical. This could be explained by the finding that mothers spend more time together with their preschool children than fathers, who are primarily responsible for the financial security of their families and are more often working in fulltime occupations/jobs (Craig, 2006; Sigmund, Badura, et al., 2016).

In contrast to the different weekday-weekend PA patterns among family members with pre-schoolers varying by body weight, weekday-weekend ST patterns were almost the same for all the members of the families, independently of the pre-schoolers' weight status. In accordance with other studies of parental proxy-reported sedentary behaviour (Cardon et al., 2016; De Craemer, Lateva, et al., 2015; Jago et al., 2014; Kourlaba, Kondaki, Liarigkovinos, \& Manios, 2009), the Czech preschool children and their parents, regardless of gender and body weight level, showed higher ST at weekends than on weekdays.

However, the Czech pre-schoolers in our sample showed less daily ST compared with the US (Mendoza et al., 2007) and European pre-schoolers from Belgium, Bulgaria, Germany, Greece, Poland, and Spain (Cardon et al., 2016; De Craemer, Lateva, et al., 2015; Jago et al., 2014; Kourlaba et al., 2009) both on weekdays and at weekends. More specifically, the parents' proxyreported daily ST of Czech pre-schoolers on weekdays is lower by more than 20 minutes than the mean ST of the pre-schoolers from the above-mentioned six European countries (normal body weight: $51.7_{\mathrm{CZECH}}$ minutes/day vs. $76.3_{\text {EUROPE }}$ minutes/day; overweight/obese: 49. $7_{\text {CZECH }}$ minutes/day vs. $88.9_{\text {EUROPE }}$ minutes/day). At weekends, the daily ST difference between the Czech and European pre-schoolers even exceeds 50 minutes a day (normal body weight: $79.6_{\mathrm{CZECH}}$ minutes/day vs. $134.0_{\text {EUROPE }}$ minutes/day; overweight/obese: $77.3_{\text {CZECH }}$ minutes/day vs. $150.2_{\text {EUROPE }}$ minutes/day [Cardon et al., 2016]).

Accordingly, at weekends a greater percentage of the children (as well as mothers and fathers) exceeded 1 hour (and 2 hours, respectively) of ST a day than on weekdays. In addition, at weekends the mothers' and fathers' ST correlated with the children's ST (as did their step counts) more strongly than on weekdays (Sigmund, Badura, et al., 2016). However, joint family time at weekends is mostly spent more passively than on weekdays, when children spend a considerable part of a day in kindergarten (Sigmundová et al., 2016). Nevertheless, when a mother achieves 10,000 steps per day at weekends, independently of the amount of parents' ST, her preschool child has higher odds (OR: 9.67, 95\% CI: $[3.57,26.23], p<.001)$ of achieving the recommended step count of 11,500 steps per day at weekends (Sigmundová et al., 2016).

When the prevalence of excessive weight of preschoolers from various European countries according to the WHO criteria (WHO Multicentre Growth Reference Study Group \& de Onis, 2006; WHO, 2007) is compared, the prevalence of overweight and obesity in Czech pre-schoolers ( $0^{\pi}: 8.5 \%_{\text {OVERWEIGHT }}$ and $9.5 \%_{\text {OBESE}}$; i: $11.4 \%_{\text {OVERWEIGHT }}$ and $9.1 \%_{\text {OBESE }}$ ) is lower compared to Spanish ( $\sigma^{\text {*: }} 29.6 \%_{\text {OVERWEIGHT }}$ and $13.4 \%_{\text {OBESE }}$; \%: $9.8 \%_{\text {OVERWEIGHT }}$ and $10.7 \%_{\text {OBESE }}$ ), Bulgarian ( $0^{x}$ : $19.3 \%_{\text {OVERWEIGHT }}$ and $7.7 \%_{\text {OBESE }}$; $+: 1.8 \%_{\text {OVERWEIGHT }}$ and $\left.7.3 \%_{\text {OBESE }}\right)$, and Polish preschool boys $\left(15.0 \%_{\text {OVERWEIGHT }}\right.$ 
and $11.8 \%_{\text {OBESE }}$ ), but higher compared to preschool children from Belgium ( $\sigma^{*}: 9.8 \%_{\text {OVERWEIGHT }}$ and $2.0 \%_{\text {OBESE }}$; ㅇ: $10.9 \%_{\text {OVERWEIGHT }}$ and $1.2 \%_{\text {OBESE }}$ ) and Germany ( $\sigma^{\prime}$ : $9.7 \%_{\text {OVERWEIGHT }}$ and $1.9 \%_{\text {OBESE }}$; $9: 7.6 \%_{\text {OVERWEIGHT }}$ and $1.7 \%_{\text {OBESE }}$ ) (van Stralen et al., 2012). Although the prevalence of overweight and obesity of the Czech pre-schoolers is at relatively low level in comparison with the pre-schoolers from other countries of Central and Eastern Europe, it is still necessary to highlight the correlates/determinants of overweight/obesity in this young age group of children to develop and improve effective and targeted obesity-related interventions.

A previous study (te Velde et al., 2012) showed strong evidence of an inverse association between PA and overweight in pre-schoolers and moderate evidence of a positive association between TV and overweight in preschool children; our results indicated a strong positive association between the excessive ST of children (>1 hour a day) and their overweight/obesity regardless of the children achieving the recommended step count of 11,500 steps/day on weekdays and at weekends. Furthermore, our results are consistent with the findings of other studies (Jago et al., 2014; Kourlaba et al., 2009; Mendoza et al., 2007), which found that one of the most important behavioural determinants of young children's ST is the ST of their parents (Jago et al., 2014). However, our study complements this finding by observing different patterns for weekdays vs. weekends. While fathers' excessive daily ST was not significantly associated with children's overweight/obesity on weekdays, mothers' excessive daily ST significantly increases the odds of overweight/obesity in their preschool children at weekends.

Our study also showed that out of all the familyrelated variables in regression models for weekdays and weekends, maternal obesity is the one most strongly associated with childhood obesity, regardless of the father's obesity. This finding is consistently reported by other studies (Durmuş et al., 2013; Sijtsma, Sauer, \& Corpeleijn, 2015), which reveal that the maternal body mass index had a stronger effect on the body mass index of preschool children than the paternal body mass index did. An analysis of the relationship between obesity in preschool children and their parents is important because parental obesity more than triples the risk of adult obesity among both obese and non-obese pre-schoolers (Whitaker et al., 1997). In addition, a higher risk of obesity in adulthood was found in preschool children with two obese parents than in preschool children with one obese parent (Whitaker et al., 1997).

\section{Strengths and limitations of the study}

Strengths of the study

The greatest strength of the present study is its monitoring of step counts as an indicator of PA and time spent watching television, video, or a computer/tablet monitor (a measure of sedentary behaviour) for all family members at the same time, which allowed for an analysis of parent-child behavioural patterns and their variations during one week. Moreover, contrary to comparable international studies (Cardon et al., 2016; De Craemer, Lateva, et al., 2015), the present study used stricter inclusion criteria (PA and ST monitoring on at least four weekdays and two weekend days for at least eight hours per day for both parents and children), which provides a more valid comparison of parent-child daily step counts and the duration of ST between weekdays and weekend days, and helps reveal the variables increasing the odds of overweight/obesity among 4-to-7-year-old Czech children.

\section{Limitations of the study}

A limitation of the study is that PA was assessed only by means of step counts. In borderline cases, an error in the measurement of step counts using the Yamax pedometer (up to 8\% [Rowe et al., 2004; Sigmund \& Sigmundová, 2014]) might have affected the classification of achieving the recommended daily number of steps in the children (De Craemer, De Decker, et al., 2015) or parents (Tudor-Locke et al., 2011). However, children's PA behaviour is considered spontaneous, highly transitory, and intermittent regardless of its intensity, with the majority of PA activity lasting up to 30 seconds (Baquet, Stratton, Van Praagh, \& Berthoin, 2007). Therefore, more accurate monitoring of PA and sedentary behaviour could be achieved by means of accelerometers or multifunctional movement devices, which, in addition to the overall amount of PA, assess the intensity, place, and course of the PA (Cerin et al., 2016; Sigmund \& Sigmundová, 2014). On the other hand, the Yamax Digiwalker SW-200 shows reasonable reliability and validity against accelerometry for children and also for adults (Cardon \& De Bourdeaudhuij, 2007; Kooiman et al., 2015). Nevertheless, to minimize the rate of rejection of the voluntary participants in the study, the above-mentioned aspect and the following aspects with a potential effect on PA, ST, and overweight/obesity among preschool children were not investigated: family socio-economic indicators (completeness of families [Kitsantas \& Gaffney, 2010]), level of parental education (Kourlaba et al., 2009), socioeconomic status (Kitsantas \& Gaffney, 2010), or parental perceptions of the safety of the neighbourhood (Cerin et al., 2016). Another possible limitation is the use of the anthropometric values of the children 
indicated by their parents. However, the applicability of this type of data for the calculation of BMI and subsequent body weight classification has been verified in the literature (Chan et al., 2013). Last, there was a difference in the sample sizes. There was a small number of participating fathers in this study and consequently also a relatively low number of whole families that provided valid data regarding all the factors that were analysed. The smaller sample could therefore have affected the results of the multivariate analyses. There were also differences in the numbers of participants between families with obese and normal-weight pre-schoolers. In particular, the number of respondents classified as obese represented a small part of our sample and this could have affected the statistical significance of our results. Last, as a result of the demands arising from the weekly monitoring of multiple family members, it might be assumed that only the 'more active' families agreed to participate in the survey. This may somewhat hinder the generalizability of our findings to all families with preschool-aged children.

\section{Conclusion}

We described different weekday-weekend patterns of PA and screen time for mothers and their preschool children. However, the odds of overweight/obesity among pre-schoolers are significantly increased by excessive screen time both on weekdays and at weekends and the mother's obesity. Moreover, at weekends, excessive screen time of mothers and children's male gender significantly increase the odds of overweight/ obesity among pre-schoolers. Therefore, weekends provide a suitable target for the implementation of family programmes aimed at reducing sedentary screen time, which is a significant factor positively affecting overweight/obesity among pre-schoolers. In sum, our findings should be a call for financially affordable family-based intervention schemes promoting active leisure time included in attractive physical activity programmes and should, as much as possible, involve both parents, together with their children.

\section{Acknowledgments}

The paper was supported by the Czech Science Foundation (grant reference number 16-14620S).

\section{Conflict of interest}

There were no conflicts of interest.

\section{References}

Aadland, E., \& Johannessen, K. (2015). Agreement of objectively measured physical activity and sedentary time in preschool children. Preventive Medicine Reports, 2, 635-639.

Australian Government, Department of Health and Ageing. (2013). Get up and grow: Healthy eating and physical activity for early childhood. Staff and carer book. Retrieved from http://www.health.gov.au/internet/main/publishing.nsf/ Content/phd-gug-staffcarers

Bandura, A. (2001). Social cognitive theory: An agentic perspective. Annual Review of Psychology, 52, 1-26.

Baquet, G., Stratton, G., Van Praagh, E., \& Berthoin, S. (2007). Improving physical activity assessment in prepubertal children with high-frequency accelerometry monitoring: A methodological issue. Preventive Medicine, 44, 143-147.

Cardon, G., \& De Bourdeaudhuij, I. (2007). Comparison of pedometer and accelerometer measures of physical activity in preschool children. Pediatric Exercise Science, 19, 205-214.

Cardon, G., De Bourdeaudhuij, I., Iotova, V., Latomme, J., Socha, P., Koletzko, B., ... ToyBox-study group. (2016). Health related behaviours in normal weight and overweight preschoolers of a large pan-European sample: The ToyBox-Study. PLoS ONE, 11, e0150580.

Cerin, E., Baranowski, T., Barnett, A., Butte, N., Hughes, S., Lee, R. E., ... O’Connor, T. M. (2016). Places where preschoolers are (in)active: An observational study on Latino preschoolers and their parents using objective measures. International Journal of Behavioral Nutrition and Physical Activity, 13, 29.

Chan, N. P. T., Choi, K. C., Nelson, E. A. S., Sung, R. Y. T., Chan, J. C. N., \& Kong, A. P. S. (2013). Self-reported body weight and height: An assessment tool for identifying children with overweight/obesity status and cardiometabolic risk factors clustering. Maternal and Child Health Journal, 17, 282-291.

Cohen, J. (1988). Statistical power analysis for the behaviour science (2nd ed.). New York, NY: Lawrence Erlbaum Associated.

Corder, K., Ekelund, U., Steele, R. M., Wareham, N. J., \& Brage, S. (2008). Assessment of physical activity in youth. Journal of Applied Physiology, 105, 977-987.

Craig, C. L., Cameron, C., \& Tudor-Locke, C. (2013). CANPLAY pedometer normative reference data for 21,271 children and 12,956 adolescents. Medicine \& Science in Sports \& Exercise, 45, 123-129.

Craig, L. (2006). Does father care mean fathers share? A comparison of how mothers and fathers in intact families spend time with children. Gender and Society, 20, 259-281.

De Craemer, M., De Decker, E., De Bourdeaudhuij, I., Verloigne, M., Manios, Y., \& Cardon, G. (2015). The translation of preschoolers' physical activity guidelines into a daily step count target. Journal of Sports Sciences, 33, 1051-1057.

De Craemer, M., Lateva, M., Iotova, V., De Decker, E., Verloigne, M., De Bourdeaudhuij, I., ... the ToyBox-study group. (2015). Differences in energy balance-related behaviours in European preschool children: The ToyBoxStudy. PLoS ONE, 10, e0118303. 
de Onis, M., Blössner, M., \& Borghi, E. (2010). Global prevalence and trends of overweight and obesity among preschool children. American Journal of Clinical Nutrition, 92, 1257-1264.

Durmuş, B., Arends, L., Ay, L., Hokken-Koelega, A., Raat, H., Hofman, A., ... Jaddoe, V. (2013). Parental anthropometrics, early growth and the risk of overweight in preschool children: The Generation R Study. Pediatric Obesity, 8, 339-350.

Huybrechts, I., Himes, J. H., Ottevaere, C., De Vriendt, T., De Keyzer, W., Cox, B., ... De Henauw, S. (2011). Validity of parent-reported weight and height of preschool children measured at home or estimated without home measurement: A validation study. BMC Pediatrics, 11, 63.

Jago, R., Thompson, J., Sebire, S., Wood, L., Pool, L., Zahra, J., \& Lawlor, D. (2014). Cross-sectional associations between the screen-time of parents and young children: Differences by parent and child gender and day of the week. International Journal of Behavioral Nutrition and Physical Activity, 11, 54.

Katzmarzyk, P., Barreira, T., Broyles, S., Champagne, C., Chaput, J.-P., Fogelholm, M., ... Church, T. (2013). The International Study of Childhood Obesity, Lifestyle and the Environment (ISCOLE): Design and methods. BMC Public Health, 13, 900.

Kelly, P., Fitzsimons, C., \& Baker, G. (2016). Should we reframe how we think about physical activity and sedentary behaviour measurement? Validity and reliability reconsidered. International Journal of Behavioral Nutrition and Physical Activity, 13, 32.

Kitsantas, P., \& Gaffney, K. F. (2010). Risk profiles for overweight/obesity among preschoolers. Early Human Development, 86, 563-568.

Kooiman, T. J. M., Dontje, M. L., Sprenger, S. R., Krijnen, W. P., van der Schans, C. P., \& de Groot, M. (2015). Reliability and validity of ten consumer activity trackers. $B M C$ Sports Science, Medicine and Rehabilitation, 7, 24.

Kourlaba, G., Kondaki, K., Liarigkovinos, T., \& Manios, Y. (2009). Factors associated with television viewing time in toddlers and preschoolers in Greece: The GENESIS study. Journal of Public Health, 31, 222-230.

Mendoza, J. A., Zimmerman, F. J., \& Christakis, D. A. (2007). Television viewing, computer use, obesity, and adiposity in US preschool children. International Journal of Behavioral Nutrition and Physical Activity, 4, 44.

Oliver, M., Schofield, G. M., Kolt, G. S., \& Schluter, P. J. (2007). Pedometer accuracy in physical activity assessment of preschool children. Journal of Science and Medicine in Sport, 10, 303-310.

Parikka, S., Mäki, P., Levälahti, E., Lehtinen-Jacks, S., Martelin, T., \& Laatikainen, T. (2015). Associations between parental BMI, socioeconomic factors, family structure and overweight in Finnish children: A path model approach. BMC Public Health, 15, 271.

Rowe, D. A., Mahar, M. T., Raedeke, T. D., \& Lore, J. (2004). Measuring physical activity in children with pedometers: Reliability, reactivity, and replacement of missing data. Pediatric Exercise Science, 16, 343-354.

Salmon, J., Campbell, K. J., \& Crawford, D. A. (2006). Television viewing habits associated with obesity risk factors:
A survey of Melbourne schoolchildren. Medical Journal of Australia, 184, 64-67.

Sigmund, E., Badura, P., Vokacova, J., \& Sigmundová, D. (2016). Parent-child relationship of pedometer-assessed physical activity and proxy-reported screen time in Czech families with preschoolers. International Journal of Environmental Research and Public Health, 13, 740.

Sigmund, E., \& Sigmundová, D. (2014). School-related physical activity, lifestyle and obesity in children. Olomouc, Czech Republic: Palacky University Olomouc.

Sigmund, E., Sigmundová, D., Badura, P., Trhlíková, L., \& Madarasová Gecková, A. (2016). Time trends: A ten-year comparison (2005-2015) of pedometer-determined physical activity and obesity in Czech preschool children. BMC Public Health, 16, 560.

Sigmundová, D., Sigmund, E., Badura, P., Vokáčová, J., Trhlíková, L., \& Bucksch, J. (2016). Weekday-weekend patterns of physical activity and screen time in parents and their pre-schoolers. BMC Public Health, 16, 898.

Sijtsma, A., Sauer, P. J., \& Corpeleijn, E. (2015). Parental correlations of physical activity and body mass index in young children- the GECKO Drenthe cohort. International Journal of Behavioral Nutrition and Physical Activity, 12, 132.

te Velde, S., Van Nassau, F., Uijtdewilligen, L., Van Stralen, M., Cardon, G., De Craemer, M., ... Chinapaw, M. (2012). Energy balance-related behaviours associated with overweight and obesity in preschool children: A systematic review of prospective studies. Obesity Reviews, 13(Suppl. 1), 56-74.

Tremblay, M. S., Colley, R. C., Saunders, T. J., Healy, G. N., \& Owen, N. (2010). Physiological and health implications of a sedentary lifestyle. Applied Physiology, Nutrition, and Metabolism, 35, 725-740.

Tudor-Locke, C., Craig, C., Brown, W., Clemes, S., De Cocker, K., Giles-Corti, B., ... Blair, S. (2011). How many steps/day are enough? For adults. International Journal of Behavioral Nutrition and Physical Activity, 8, 79.

van Stralen, M. M., te Velde, S. J., van Nassau, F., Brug, J., Grammatikaki, E., Maes, L., ... Chinapaw, M. J. (2012). Weight status of European preschool children and associations with family demographics and energy balance-related behaviours: A pooled analysis of six European studies. Obesity Reviews, 13(Suppl. 1), 29-41.

Whitaker, R. C., Wright, J. A., Pepe , M. S., Seidel, K. D., \& Dietz, W. H. (1997). Predicting obesity in young adulthood from childhood and parental obesity. New England Journal of Medicine, 337, 869-873.

WHO Multicentre Growth Reference Study Group, \& de Onis, M. (2006). WHO Child Growth Standards based on length/height, weight and age. Acta Pcediatrica, 95(Suppl. S450), 76-85.

World Health Organization. (2007). Growth reference data for 5-19 years. Retrieved from http://www.who.int/growthref/ en/

World Health Organization. (2014). Obesity and overweight. Fact sheet No 311. Retrieved from http://www.who.int/ mediacentre/factsheets/fs311/en/ 